\title{
Flora das cangas da Serra dos Carajás, Pará, Brasil: Hypnaceae
}

\author{
Flora of the canga of the Serra dos Carajás, Pará, Brazil: Hypnaceae
}

Fúvio Rubens Oliveira-da-Silva ${ }^{1} \&$ Anna Luiza Ilkiu-Borges ${ }^{1,2}$

\begin{abstract}
Resumo
Foram registradas três espécies de Hypnaceae (Chryso-hypnum diminutivum, Ectropothecium leptochaeton e Rhacopilopsis trinitensis) nas áreas de canga na Serra dos Carajás, no estado do Pará, para as quais se apresenta descrição, ilustração e comentários morfológicos.

Palavras-chave: Brioflora, FLONA Carajás, musgos, taxonomia.
\end{abstract}

\begin{abstract}
Three species of Hypnaceae (Chryso-hypnum diminutivum, Ectropothecium leptochaeton and Rhacopilopsis trinitensis) were recorded in the areas of canga in the Serra dos Carajás, Pará state, for which presented descriptions, illustrations, and morphological comments.
\end{abstract}

Key words: Bryoflora, FLONA Carajás, mosses, taxonomy.

\section{Hypnaceae}

Hypnaceae Schimp. reúne 66 gêneros (Goffinet et al. 2009) de musgos pleurocárpicos, dos quais apenas 12 (com 28 espécies) ocorrem no Brasil (Costa \& Peralta 2015). A família é caracterizada pelo caulídio rastejante ou ascendente, regular ou irregularmente pinado ou bipinado, pseudoparáfilos folhosos ou filamentosos, filídios tipicamente homômalos a falcado-secundos, costa curta e dupla ou ausente, células dos filídios lineares, cápsulas inclinadas e assimétricas, e células alares não diferenciadas (Gradstein et al. 2001; Goffinet et al. 2009). Nas cangas da Serra dos Carajás foram registrados os gêneros: Chryso-hypnum Hampe, Ectropothecium Mitt. e Rhacopilopsis Renauld \& Cardot.

\section{Chave de identificação dos gêneros de Hypnaceae das cangas da Serra dos Carajás}

1. Filídios dimórficos (diferenciados no mesmo ramo)

3. Rhacopilopsis

1'. Filídios monomórficos

2. Filídios eretos a estendidos. Células conspicuamente prorulosas. Ápice dos filídios acuminado .

1. Chryso-hypnum

2'. Filídios eretos a falcados ou falcado-secundos. Células lisas (nunca prorulosas). Ápice dos filídios curto- a longo-acuminado

2. Ectropothecium

\section{Chryso-hypnum Hampe}

$\mathrm{O}$ gênero apresenta cinco espécies na região neotropical e duas espécies no Brasil (Gradstein et al. 2001; Costa \& Peralta 2015). Ocorrem geralmente sobre solo, troncos de árvores e rochas, em ambientes úmidos e semi-secos (Gradstein et al.
2001). As espécies deste grupo são caracterizadas pelas plantas com filídios monomórficos, ovaladolanceolados, ápice acuminado, costa dupla e curta, pseudoparáfilos folhosos, células conspicuamente prorulosas na extremidade superior (Gradstein et al. 2001; Buck 1998; 2003).

\footnotetext{
${ }^{1}$ Museu Paraense Emílio Goeldi, Av. Magalhães Barata 376, São Braz, 66040-170, Belém, PA, Brasil.

${ }^{2}$ Autor para correspondência: ilkiu-borges@museu-goeldi.br
} 
1.1. Chryso-hypnum diminutivum (Hampe) W.R. Buck, Brittonia 36: 182. 1984.

Hypnum diminutivum Hampe, Linnaea 20(1): 86-87. 1847.

Fig. 1a-c

Plantas verde-pálidas a esbranquiçadas. Ramos subpinados, prostrados ou ascendentes, curtos, filídios adensados, às vezes complanadofoliados. Filídios dos ramos principal e secundários pouco diferenciados, eretos a estendidos, ovalados a ovalado-lanceolados, $0,7-1 \times 0,2-0,3 \mathrm{~mm}$, ápice acuminado, margem serrilhada, plana, costa dupla, de tamanho desigual, 1/4-1/3 do comprimento do filídio. Células lineares, $37,5-50 \times 2,5-7,5 \mu \mathrm{m}$, prorulosas na extremidade superior (visível principalmente na parte dorsal). Células alares pouco diferenciadas, ca. 12 células subquadradas, $10-15 \mu \mathrm{m}$.

Material selecionado: Canaã dos Carajás, S11B, 6²1'19,1'S, 50²3'27,4”'W, 29.IV.2015, A.L. IlkiuBorges et al. 3518 (MG).

Chryso-hypnum diminutivum é caracterizado pelos filídios ovalados a ovaladolanceolados, costa dupla e desigual, alcançando de 1/4 a 1/3 do comprimento do filídio, células prorulosas na extremidade superior e células alares quadráticas pouco diferenciadas. De acordo com Buck (1998, 2003), os filídios do ramo principal e dos ramos secundários se diferenciam pouco no tamanho e na disposição no caulídio, sendo os do ramo principal um pouco maiores e variando de eretos a amplamente estendidos, e os dos ramos secundários menores e amplamente estendidos.

Cresce geralmente sobre madeira em decomposição (Buck 1998, 2003). Na Serra dos Carajás, essa espécie ocorreu sobre tronco morto em mata baixa sobre canga.

Pantropical. No Brasil: AC, AM, AP, DF, ES, GO, MG, MT, PA, PE, PR, RJ, RR, RS, SC, SP. Serra dos Carajás: Serra Sul: S11B.

\section{Ectropothecium Mitt.}

Este é um gênero pantropical com mais de 200 espécies de maior diversidade na Ásia, enquanto na região neotropical, ocorrem ca. de cinco espécies (Gradstein et al. 2001). No Brasil, são reconhecidas duas espécies (Costa \& Peralta 2015). Ocorrem sobre solo, húmus e troncos em decomposição (Gradstein et al. 2001). O gênero é caracterizado pelos filídios monomórficos, falcados a falcado-secundos, às vezes eretos, com ápice curto- a longo-acuminado, costa dupla ou ausente, pseudoparáfilos folhosos ou filamentosos, células do filídios lisas (Gradstein et al. 2001; Buck 1998; 2003).

2.1. Ectropothecium leptochaeton (Schwägr.) W.R. Buck, Brittonia 35: 311. 1983.

Hypnum leptochaeton Schwägr. Species muscorum frondosorum, Supplementum Primum 2: 296.1816.

Fig. 1d-g

Plantas verdes a marrom-claras. Ramos regularmente e densamente pinados, curtos. Filídios dos ramos principal e secundários pouco diferenciados, eretos a falcados ou falcados-secundos, lanceolados a ovaladolanceolados, $0,7-1,2 \times 0,2-0,4 \mathrm{~mm}$, ápice curtoa longo-acuminado, geralmente enrolados e torcidos, margem levemente serrilhada, plana a irregularmente curvada, costa ausente. Células lineares a linear-flexuosas, $60-125 \times 2,5-5 \mu \mathrm{m}$, lisas. Células alares pouco diferenciadas, 1-3 células subquadradas, 10-25 $\mu \mathrm{m}$.

Material examinado: Parauapebas, N1, 28.III.1993, C.S. Rosário \& J.S. Ramos 630 (MG); N2, 31.III.1993, C.S. Rosário \& J.S. Ramos 818 (MG).

De acordo com Buck (2003), os filídios do ramo principal e dos ramos secundários se diferenciam no tamanho e na forma. Enquanto os filídios do ramo principal são maiores $(0,8-1,2$ $\mathrm{mm}$ de comprimento) e ovalado-lanceolados, os dos ramos secundários são um pouco menores $(0,6-0,9 \mathrm{~mm}$ de comprimento) e lanceolados a oblongo-lanceolados.

Segundo Gradstein et al. (2001) e Buck (2003), essa espécie é a mais comum e amplamente distribuída na região neotropical, crescendo geralmente sobre solo e ocasionalmente sobre rochas, base de árvores e madeira em decomposição. Nas cangas da Serra dos Carajás, essa espécie ocorreu sobre madeira em decomposição.

Neotropical. No Brasil: AM, ES, MG, MS, MT, PA, PR, RJ, SC. Serra dos Carajás: Serra Norte: N1 e N2.

\section{Rhacopilopsis Renauld \& Cardot.}

$\mathrm{O}$ gênero contém cinco espécies de maior distribuição na África tropical. No Neotrópico, contém uma espécie que ocorre preferencialmente sobre troncos e casca de árvores (Gradstein et al. 2001). Os membros desse grupo são identificados pelos filídios dimórficos (no mesmo ramo), filídios laterais e dorsais grandes e assimétricos, filídios ventrais pequenos e simétricos, costa 

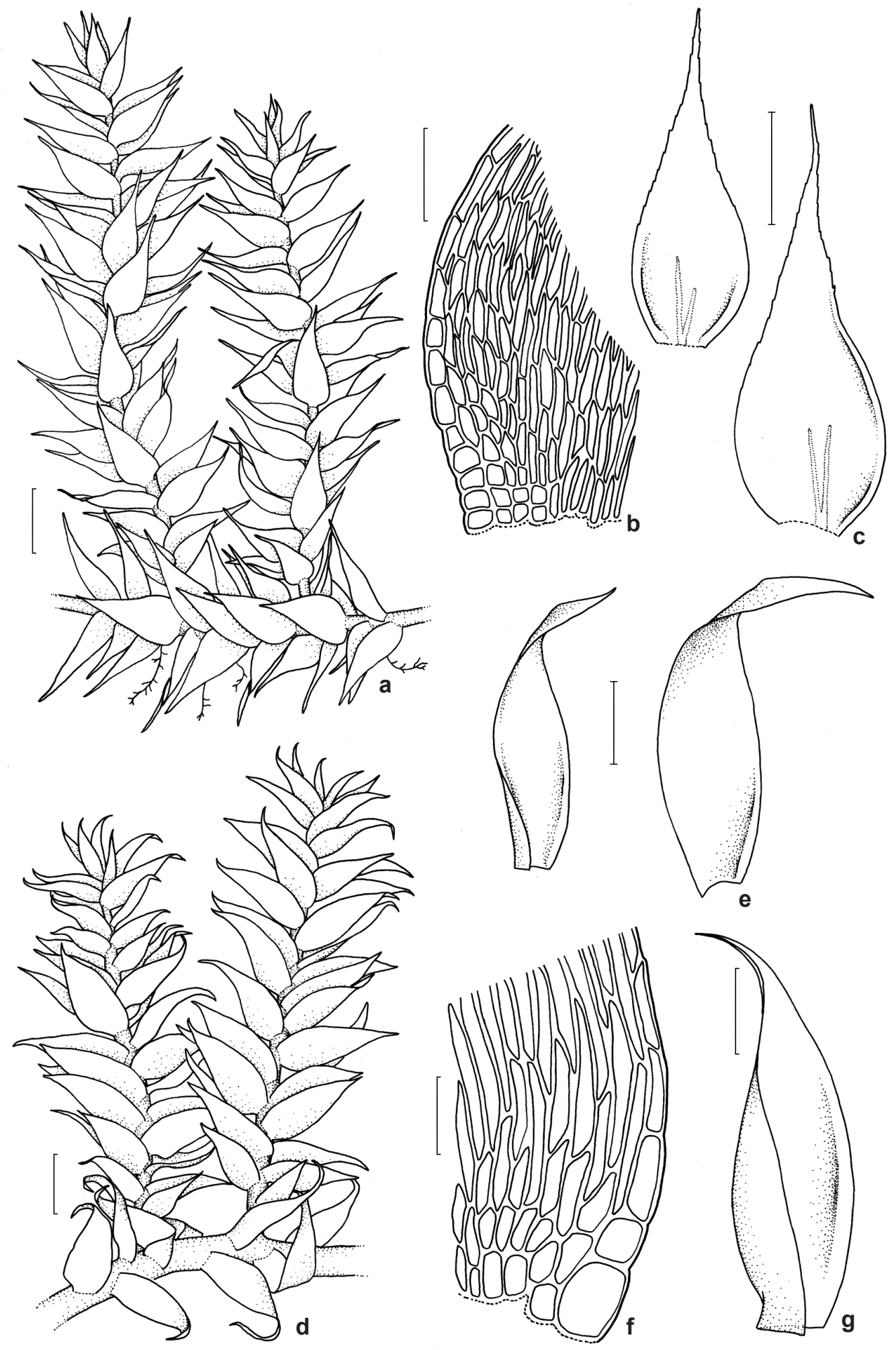

Figura 1 -a-c. Chryso-hypnum diminutivum - a. hábito; b. base do filídio; c. filídios. d-g. Ectropothecium leptochaeton - d. hábito; e. filídios; f. base do filídio; g. filídio. Barras: a, $d=500 \mu \mathrm{m}$; c, e, $g=250 \mu \mathrm{m} ; \mathrm{b}=50 \mu \mathrm{m}, \mathrm{f}=25 \mu \mathrm{m}$.

Figure 1 - a-c. Chryso-hypnum diminutivum - a. habit; b. leaf base; c. leaves. d-g. Ectropothecium leptochaeton - d. habit; e. leaves; f. leaf base; g. leaf. Bars: $\mathrm{a}, \mathrm{d}=500 \mu \mathrm{m} ; \mathrm{c}, \mathrm{e}, \mathrm{g}=250 \mu \mathrm{m} ; \mathrm{b}=50 \mu \mathrm{m}, \mathrm{f}=25 \mu \mathrm{m}$. 
dupla, curta, pseudoparáfilos filamentosos, células do filídio lisas (Gradstein et al. 2001; Buck 1998, 2003).

3.1. Rhacopilopsis trinitensis (Müll. Hal.) E. Britt. \& Dixon, J. Bot. British and Foreign 60: 88. 1927. Hypnum trinitense Müll. Hal. Synopsis muscorum frondosorum omnium hucusque cognitorum 2: 284. 1851.

Fig. 2a-f
Plantas amarelo-pálidas. Ramos regularmente pinados, prostrados ou ascendentes, densamente e irregularmente foliados. Filídios do ramo principal e dos ramos secundários dimórficos (ventrais e laterais diferenciados), filídios laterais assimétricos, oblongo-ovalados a cultriformes, côncavos, $0,7-1$ $\times 0,2-0,3 \mathrm{~mm}$, ápice longo-acuminado, filídios ventrais simétricos, ovalado-lanceolados, \pm planos, $0,3-0,7 \times 0,1-0,2 \mathrm{~mm}$, ápice curto- a longo-
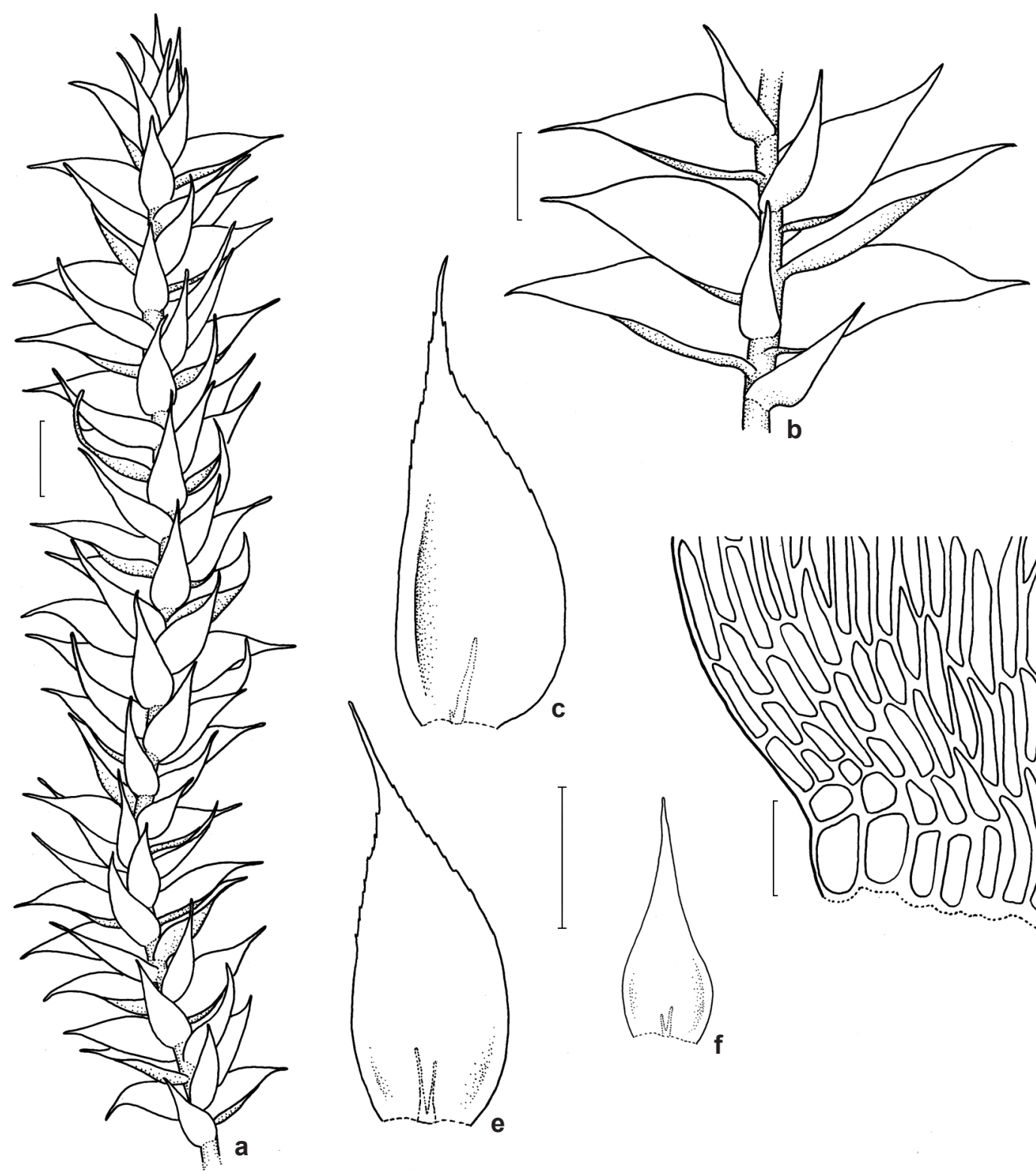

Figura 2 - a-f. Rhacopilopsis trinitensis - a-b. hábito; c. filídio lateral; d. base do filídio; e. filídio lateral; f. filídio ventral. Barras: $\mathrm{a}=500 \mu \mathrm{m} ; \mathrm{b}, \mathrm{c}, \mathrm{e}, \mathrm{f}=250 \mu \mathrm{m} ; \mathrm{d}=25 \mu \mathrm{m}$.

Figure 2 - a-f. Rhacopilopsis trinitensis - a-b. habit; c. lateral leaf; d. leaf base; e. lateral leaf; f. ventral leaf. Bars: $a=500 \mu \mathrm{m} ; \mathrm{b}, \mathrm{c}$, $\mathrm{e}, \mathrm{f}=250 \mu \mathrm{m} ; \mathrm{d}=25 \mu \mathrm{m}$. 
acuminado. Margem dos filídios serrilhadas, costa dupla, desiguais, até $1 / 4$ do comprimento do filídio. Células medianas longo-hexagonais a lineares, $30-70 \times 3-8 \mu \mathrm{m}$, lisas. Células alares pouco diferenciadas, geralmente de 3-4 células subquadradas, $10-20 \mu \mathrm{m}$.

Material selecionado: Parauapebas, N1, 28.III.1993, C.S. Rosário \& J.S. Ramos 639 (MG); N2, 31.III.1993, C.S. Rosário \& J.S. Ramos 835 (MG); N5, 606'18,1"S, 5007'49,3”'W, 27.IV.2015, A.L. Ilkiu-Borges et al. 3391 (MG).

Os filídios diferenciados no mesmo ramo (dimórficos), costa dupla até $1 / 4$ do comprimento do filídio e células lisas longo-hexagonais a lineares são as principais características para a identificação desta espécie.

Segundo Buck (1998), Rhacopilopsis trinitensis cresce geralmente sobre troncos de árvores, especialmente na base. Nas cangas da Serra dos Carajás, a espécie ocorreu sobre árvore viva, rocha de ferro e solo.

Pantropical. No Brasil: AL, AM, AP, MG, MS, MT, PA, PE, RR, SP. Serra dos Carajás: Serra Norte: N1, N2 e N

\section{Agradecimentos}

Agradecemos ao Museu Paraense Emílio Goeldi e ao Instituto Tecnológico Vale, a infraestrutura e demais apoios fundamentais para o desenvolvimento deste trabalho, assim como à Dra. Ana Maria Giulietti Harley e ao Dr. Pedro Viana, coordenadores do projeto conveniado MPEG/ITV/FADESP (01205.000250/2014-10) e ao projeto aprovado pelo $\mathrm{CNPq}$ (processo 455505/2014-4), o financiamento; ao ICMBio, em especial ao biólogo Frederico Drumond Martins, a licença de coleta concedida e suporte nos trabalhos de campo; ao CNPq, a bolsa de Mestrado concedida ao primeiro autor e a bolsa de Produtividade em Pesquisa concedida à segunda autora.

\section{Referências}

Buck WR (1998) Pleurocarpous mosses of the West Indies. Memoirs of The New York Botanical Garden 82: 1-400.

Buck WR (2003) Guide to the plants of Central French Guiana. Part 3. Mosses. Memoirs of The New York Botanical Garden 76: 1-167.

Costa DP \& Peralta DF (2015) Bryophytes diversity in Brazil. Rodriguésia, 66: 1063-1071.

Goffinet B, Buck WR \& Shaw AJ (2009) Morphology and classification of the Bryophyta. In: Goffinet B \& Shaw AJ (eds.) Bryophyte Biology. Cambridge University Press, Cambridge. Pp. 55-138.

Gradstein SR, Churchill SP \& Salazar-Allen N (2001) Guide to the Bryophytes of Tropical America. Memoirs of the New York Botanical Garden 86: 1-577.

\section{Lista de exsicatas}

Ilkiu-Borges AL et al. 3391 (3.1), 3518 (1.1). Rosário CS \& Ramos JS 816 (2.1), 862 (2.1), 630 (2.1), 639 (3.1), 814 (3.1), 815 (3.1), 818 (2.1), 823 (3.1), 835 (3.1), 837 (3.1), 864 (3.1), 878 (3.1). 
